\title{
LA JUSTIFICACIÓN DEL DERECHO DE RESISTENCIA EN EL ESTADO CONSTITUCIONAL DEMOCRÁTICO DE DERECHO: ALGUNAS REFLEXIONES IUSFILOSÓFICAS
}

\author{
Eduardo Esteban Magoja \\ Facultad de Derecho \\ Universidad de Buenos Aires
}

$\underline{\text { http://dx.doi.org/10.5209/rev NOMA.2016.v47.n1.52395 }}$

\begin{abstract}
Resumen.- Este trabajo tiene el propósito de demostrar cómo en el Estado Constitucional de Derecho el derecho de resistencia tiene un papel clave para su desarrollo, su adecuación a la realidad cambiante de la sociedad y la satisfacción de los intereses de todos los involucrados en ese proyecto común. En primer lugar, analizaremos cómo deben actuar los individuos o los grupos sociales que sufran injusticias a causa de actos estatales o leyes que violen sus derechos más elementales. En ciertos casos, creemos que tienen el derecho a ejercer cualquier forma de resistencia débil que consideren apropiada para presentar en la escena pública una causa que debe ser social y políticamente reconocida. En segundo lugar, veremos qué sucede cuando se llega a la instancia de que es el propio Estado de Derecho aquel que se encuentra en peligro. En ese caso, creemos que la sociedad no sólo tendrá el derecho, sino el deber de ejercer la resistencia en su forma más extrema para defender el orden constitucional vigente de cualquier autoridad ilegítima que pretenda imponerse sobre él y la soberanía del pueblo.
\end{abstract}

Palabras clave: derecho de resistencia, injusticia, Estado de Derecho, bienes humanos básicos

\begin{abstract}
This paper aims to demonstrate how in the constitutional rule of law the right of resistance plays a key role in its development, its adaptation to the changing reality of society and the satisfaction of the interests of all the people involved in this common project. Firstly, we will analyze how individuals or social groups must act when they suffer injustices due to state acts or laws that violate their most basic rights. In some cases, we believe that they have the right to exercise any form of weak resistance that they deem appropriate to present at the public scene a cause that must be socially and politically recognized. Secondly, we will see what happens when the rule of law itself is in danger. In that case, we believe that society will have not only the right but the duty to exercise the resistance in its most extreme form to defend the existing constitutional order of any illegitimate authority that seeks to impose itself on it and the sovereignty of the people.
\end{abstract}

Keywords: right of resistance, injustice, rule of law, basic human goods

\section{Introducción}

Generalmente se dice que el derecho debe ser obedecido. Sin embargo, la pregunta acerca de si debe obedecérselo no tiene una respuesta sencilla. En primer lugar, depende de qué se entienda por derecho. Si, por ejemplo, concebimos el derecho como una técnica social específica que permite promover la cooperación y asegurar la paz social ${ }^{1}$, se puede decir que la

\footnotetext{
${ }^{1}$ Cf. Kelsen (1949: 22-23); Ross (1961: 130); Raz (1982: 144 y ss.). Así, Raz (1999: 159-160) sostiene que las funciones del derecho relativas a la coordinación, resolución de conflictos y reparación de daños son necesarias incluso en una sociedad de ángeles.
} 
obediencia se vuelve un principio sumamente importante. Si, en cambio, sostenemos, desde una visión marxista, que el derecho es un instrumento de dominación de una clase y sus intereses sobre otra clase y sus intereses, que al mismo tiempo enmascara la explotación capitalista y acentúa las desigualdades $^{2}$, el panorama sería otro: siempre y cuando no se quiera justificar el sometimiento de las clases sociales menos aventajas a las clases que detentan los medios de producción, la obediencia debería ser rechazada.

En segundo lugar, aunque consideráramos que el derecho sea un instrumento que aspira a la paz, que es la visión que compartimos, esto no significa que siempre se deba obedecerlo. No podemos hacer de la obediencia un principio absoluto porque pueden existir ciertas leyes que sean claramente injustas o que entren en conflicto con valores socialmente superiores como la libertad o la igualdad. En estos casos, si se quiere preservar la dignidad y la integridad moral del hombre no se debería obedecer, sino tomar otra postura.

Resulta evidente, entonces, que la pregunta acerca de si debe obedecerse el derecho tiene varias facetas. No existe una sola posición del individuo o el cuerpo cívico con respecto a la autoridad, sino que su conducta, como explica Bobbio (1997: 118-119), puede oscilar desde el aval total del orden legal hasta la completa rebeldía. Esto nos conecta con una tercera cuestión. Junto a la pregunta acerca de si debe obedecerse el derecho se sitúa la pregunta acerca de si debe desobedecérselo. Ambas cuestiones son dos caras de la misma moneda.

Ahora bien, en este trabajo nos detendremos en analizar este último punto y realizar algunas consideraciones filosóficas. No nos interesa, por cierto, estudiar cualquier tipo de desobediencia, sino una que tiene especial interés dentro de la filosofía política y jurídica: la desobediencia a través del ejercicio del derecho de resistencia lato sensu. La tesis que se defenderá aquí es que, si bien en el Estado Constitucional de Derecho las leyes deben ser obedecidas, la resistencia constituye una herramienta imprescindible en su perfeccionamiento. Se buscará ofrecer una justificar acerca de cómo ella, más que como una actitud transgresora, debe pensarse como una acción constructiva y defensora del orden constitucional y los derechos humanos básicos.

\section{El derecho de resistencia en la historia: la lucha del hombre contra las injusticias y las formas de opresión estatal.}

Más allá de su ambigüedad conceptual, el derecho de resistencia lato sensu comprende todo el abanico de conductas cuyo denominador común es el

\footnotetext{
${ }^{2}$ En Marx, el derecho contribuye al mantenimiento y desarrollo de una forma de producción, sometiendo a la clase económicamente explotada y orientando su actividad a favor de las relaciones económicas existentes. Resulta elocuente, al respecto, el siguiente pasaje de Marx \& Engels (1974 [1846]: 430) de La ideología alemana: "las relaciones de producción de los individuos que hasta ahora han venido dominando no tienen más remedio que manifestarse también en el plano de las relaciones políticas y jurídicas". Así también se pronuncia Gramsci (1974: 162), quien sostiene que "el derecho no expresa a toda la sociedad [...], sino a la clase dirigente que 'impone' a toda la sociedad las normas de conducta que están más ligadas a su razón de ser y a su desarrollo".
} 
enfrentamiento con el poder estatal, ya sea para cuestionar su legitimidad o bien la justicia de su actuación en casos puntuales ${ }^{3}$. La importancia de ese derecho atraviesa toda la historia del hombre ${ }^{4}$. Una de las primeras reflexiones acerca de él se puede ver ya en Grecia, en especial en Antígona de Sófocles (c. 442 a. C.). Sus primeras formulaciones teóricas tendrán lugar en la Edad Media de la mano de figuras como Juan de Salisbury, Tomas de Aquino y Ockham, y en la Modernidad ocupará el foco de atención de autores como Suárez, Lutero, Calvino, Althusius, Grocio, Pufendorf, Leibniz, Thomasius, entre muchos otros.

En virtud del ejercicio de la resistencia el hombre fue ganando el reconocimiento de derechos básicos que consideraba imprescindibles para el desarrollo de la vida: desde la libertad, la igualdad, hasta los derechos económicos, sociales y culturales. Los diversos instrumentos jurídicos que fueron reconociendo los derechos humanos son testigos del proceso: la Carta Magna de Inglaterra de 1215, la Petición de Derechos de 1628, el Acta de Habeas Corpus de 1679, la Declaración de Derechos de 1689, la Declaración de Independencia de los Estados Unidos de 1776, la Declaración de los Derechos del Hombre y del Ciudadano de 1789, la Declaración Universal de Derechos Humanos de 1948 y el Pacto Internacional de Derechos Civiles y Políticos de 1966, entre otros instrumentos internacionales. Todos ellos son conquistas o -utilizando la expresión de Dworkin (1984: 37)- "triunfos políticos" que el hombre obtuvo a través de la lucha contra las injusticia y las diversas formas de opresión estatal.

La importancia que tuvo el derecho de resistencia en la defensa y protección de los bienes humanos básicos se percibe en su proceso de positivización. En efecto, este derecho fue reconocido por primera vez en la Constitución de Florencia del año 1508 y alcanzó su máxima expresión en la modernidad con la Declaración de Independencia de los Estados Unidos de 1776 y la Declaración de los Derechos del Hombre y del Ciudadano de 1789. En ambas declaraciones el reconocimiento declarativo del derecho de resistencia tiene como fuente más significativa la filosofía de Locke ${ }^{5}$. En su magna obra The Second Teatise of Civil Goverment de 1689 el filósofo destacó tres ideales esenciales: el carácter inalienable de ciertos derechos básicos; el consenso de los gobernados como elemento esencial de la autoridad legítima; la obligación primaria del gobierno de proteger esos derechos inalienables. En ese contexto agregó, como cuarta idea, que el pueblo puede legítimamente resistir y derrocar a aquel gobierno que no respetara aquellos derechos básicos ${ }^{6}$.

Es notable como todos estos principios son trasladas a las declaraciones. En el caso de la Declaración de Estados Unidos se afirmó, como verdades evidentes, que "todos los hombres son creados iguales; que son dotados por su Creador de ciertos derechos inalienables; que entre éstos están la vida, la libertad y la búsqueda de la felicidad; que para garantizar estos derechos se instituyen

\footnotetext{
${ }^{3}$ Cf. Ugartemendia (1999: 214).

${ }^{4}$ Con respecto a la historia del derecho de resistencia ver Carvajal (1991), Ugartemendia (1999).

${ }^{5}$ Cf. Gargarella (2007: 5); Ugartemendia (1999: 223).

${ }^{6}$ Ver, en especial, los $\S \S 221$ y ss.
} 
entre los hombres los gobiernos, que derivan sus poderes legítimos del consentimiento de los gobernados; que cuando quiera que una forma de gobierno se haga destructora de estos principios, el pueblo tiene el derecho a reformarla o abolirla e instituir un nuevo gobierno que se funde en dichos principios, y a organizar sus poderes en la forma que a su juicio ofrecerá las mayores probabilidades de alcanzar su seguridad y felicidad".

En el caso de la Declaración francesa, estas ideas se recogieron en los artículos 1 y 2: mientras que en el primero de ellos se dice que los "hombres nacen y permanecen libres e iguales en derechos", en el otro se afirma que "la finalidad de cualquier asociación política es la protección de los derechos naturales e imprescriptibles del Hombre", que son "la libertad, la propiedad, la seguridad y la resistencia a la opresión".

La Declaración de Estados Unidos y la Declaración de Francia son dos instrumentos que nacieron en el marco de revoluciones distintas; sin embargo, en ellos se expresa una idea común sobre la cual conviene insistir: el derecho de resistencia como elemento clave en la protección y defensa de los derechos básicos del hombre frente a los abusos de la autoridad estatal. Este derecho, por cierto, no quedó acotado al ámbito de esas declaraciones, sino que también fue recogido en varias Constituciones que surgieron de la atmosfera generada por las revoluciones americana y francesa. A modo de ejemplo, podemos nombrar la Constitución de Francia de 1793 y, en Latinoamérica, el Proyecto de Constitución de la Banda Oriental de 1813 y la Constitución de Apatzingán de 1814.

En el siglo $X X$, luego de que finalizaran los terribles sucesos de la Segunda Guerra Mundial, el derecho de resistencia adquirió una nueva etapa en la cual se integró al derecho constitucional contemporáneo y al nuevo orden internacional de los Derechos Humanos ${ }^{7}$. En Europa, cabe destacar el Proyecto de Constitución de la República de Italia de 1947 (artículo 50) y, en especial, la Constitución de la República Federal de Alemania de 1949 (artículo 20, inc. 4), cuyo texto aún está vigente. En América Latina, el derecho de resistencia se verá plasmado en las constituciones de Cuba (artículo 3), Honduras (artículo 3), Ecuador (artículo 98) y Venezuela (artículo 350), entre otros. En Argentina, en especial, ese derecho se incorporó en la Constitución Nacional, mediante su reforma en 1994, como respuesta al proceso de desconstitucionalización que vivió entre 1930 y 1983 a causas de los gobiernos militares (o cívico-militares) que se instalaban ilegítimamente en el poder ${ }^{8}$.

\section{La configuración del derecho de resistencia en el orden constitucional: algunas consideraciones en torno a su justificación}

La positivización del derecho de resistencia en las Constituciones actuales es quizás el mayor homenaje de la historia de la lucha del hombre contra los gobiernos tiránicos y los abusos de la autoridad estatal. Sin embargo, la

\footnotetext{
${ }^{7}$ Cf. Carvajal (1991: 226).

${ }^{8}$ Cf. Gelli (2008: 509).
} 
formalización del derecho origina algunos problemas teóricos sobre los cuales conviene reflexionar. En primer lugar, cabe preguntarse cómo es posible que el Estado establezca en su normativa un derecho de resistencia. Si consideramos que el derecho es sólo aquel establecido por el Estado no debería haber lugar para el derecho de resistir sus injusticias, ya que lo contrario sería aceptar que el derecho reconoce la posibilidad de ser desobedecido. Claramente esto es una contradicción ${ }^{9}$.

En segundo lugar, existe otro problema que está ligado con la validez del derecho. En efecto, si se considera como derecho válido todo aquel producido de manera correcta por el Estado, más allá de su contenido, entonces nuevamente nos encontramos con que no hay lugar para la desobediencia ${ }^{10}$. Toda conducta que se aparte de la ley será ilegal y sólo podrá estar justificada moralmente. De hecho, en este sentido se expresaba Kelsen, quien en 1968 declaró abiertamente que, desde el punto de vista de la Ciencia del Derecho, el derecho durante la dominación nazi fue derecho. Aquellos que ejercieron dentro de él la resistencia lo hicieron de una forma moralmente elogiable pero antijurídica.

Agamben (2007: 38) explica que fueron precisamente esta clase de problemas lo que llevó a rechazar la incorporación del derecho de resistencia en la actual Constitución de la República de Italia. Se consideraba imposible regular jurídicamente algo que, por su propia naturaleza, se sustrae al ámbito del derecho positivo. Está claro, entonces, que a la hora de hablar del derecho de resistencia, como dice Agamben (2007: 39) "lo que está en cuestión, en suma, es el problema del significado jurídico de una esfera de acción en sí misma extrajurídica".

Creemos que se puede encontrar una salida a este laberinto. En primer lugar, resulta necesario no identificar el derecho con el Estado y concebir el derecho de resistencia en el marco del orden constitucional al cual incluso se halla subordinada la autoridad estatal. Así pues, siguiendo la propuesta de Ugartemendia (1999: 227-231), el derecho de resistencia debe ser situado en el campo de los derechos y garantías de la Constitución Democrática, la ley suprema que gobierna el ejercicio del poder público. Esto supone que la validez del derecho de resistencia se inserta, ante todo, dentro del Estado Constitucional de Derecho. En esta concepción jurídica-política el Estado se encuentra constitucionalizado y la Constitución que lo define y determina no es axiológicamente neutral, sino que impone una serie de valoraciones que encuentran su anclaje en la protección y el seguimiento de los derechos esenciales del hombre. En este sentido, la Constitución del Estado es una estructura de principios supra-positivos que reclama validez y exige obligatoriedad.

En segundo lugar, habría que establecer una distinción del derecho mismo de resistencia. Habíamos dicho que el derecho de resistencia lato sensu puede ser ejercido para cuestionar el Estado en cuanto tal o, en cambio, la validez de algunas de sus normas jurídicas. Ambos cuestiones deben ser separadas. En

\footnotetext{
${ }^{9}$ Cf. Ugartemendia (1999: 226-227).

${ }^{10}$ Cf. Kaufmann (2006: 375).
} 
efecto, según la finalidad podríamos decir que existen grosso modo dos tipos de resistencia: una que llamaremos resistencia fuerte y otra resistencia débil. La primera de ellas engloba las acciones que rechazan una autoridad ilegítima (como por ejemplo un Estado tiránico). En cambio, la segunda categoría abarca aquellos casos en los que se cuestiona la validez de algunas normas del Estado. En esta última categoría, pues, cabe incluir todas aquellas formas de resistencia que, reconociendo la autoridad estatal como legítima, se oponen a sus actos por considerarlos arbitrarios o injustos: la desobediencia civil, la protesta y la objeción de conciencia son sus ejemplos más paradigmáticos.

La combinación de ambas precisiones nos permite tener otro panorama con respecto a los problemas planteados. La resistencia fuerte se convierte en una herramienta esencial para mantener la vigencia del orden constitucional frente a cualquiera poder ilegítimo que pretenda socavarlo: éste es, de hecho, el espíritu del artículo 36 de nuestra Constitución argentina. El derecho de resistencia no es "puesto" por el Estado, sino reconocido por la Constitución y se fundamenta en la soberanía del pueblo. Si a través de medios no establecidos por la Carta Magna se accede al poder y además se la deja sin vigencia, la soberanía sería gravemente transgredida. La violación del derecho del pueblo de elegir a sus gobernantes, imponerse sus propias leyes y exigir que se respete su voluntad, justifica que se ejerza la resistencia con el fin de reestablecer el orden constitucional democrático. Se trata, en última instancia, de un mecanismo esencial que dispone la Constitución para asegurar su continuidad $^{11}$.

La resistencia débil, aunque ilegal, vendría a constituir un vehículo imprescindible para hacer valer los derechos y garantías reconocidos en la Constitución en el caso de que sean transgredidos por el Estado (aun reconocido como legítimo), ya sea por comisión o por omisión. O incluso sirve también para que en la esfera jurídica se reconozcan derechos que emerjan de la transformación social y las nuevas luchas políticas. Más tarde volveremos en detalle sobre este tema, ya que habría que analizar un problema relevante que surge de esta propuesta. En esta instancia nos interesa insistir sobre una cuestión muy importante: la resistencia debe pensarse como una actitud constructiva y defensora del Estado Constitucional de Derecho, es decir, del Estado que bajo la órbita de la Constitución garantice, proteja y reconozca los derechos fundamentales del hombre. La resistencia que busque instaurar cualquier otro tipo de sistema político que desconozca esos derechos carece de validez. Sólo es válida la resistencia que persiga la defensa de los derechos esenciales del hombre y promueva la existencia de ordenamientos jurídicos que ubiquen como fin último al ser humano con sus valores ontológicos y existenciales.

Por lo demás, vale aclara que todo esto no significa colocar a la Constitución como un valor absoluto y omnicomprensivo, sino reconocer el papel que juega como carta de navegación de un Estado que, en principio, es aceptada y compartida en libertad y democracia por sus miembros. En última instancia, si tuviéramos que ofrecer un fundamento meta-jurídico que justifique el ejercicio

\footnotetext{
${ }^{11}$ Cf. Vitale (2009: 47).
} 
del derecho de resistencia deberían ser los derechos fundamentales del hombre, es decir, los derechos esenciales respecto de los cuales uno tiene un título simplemente por ser persona. Resulta evidente, entonces, que esos derechos pueden estar o no escritos: esto, en realidad, es una cuestión secundaria. De hecho, nuestra Constitución Nacional reconoce en el artículo 33 todos los derechos no enumerados en ella y en el Informe de la Comisión Examinadora de 1853 se señalan cuáles son: "los derechos de los hombres que nacen de su propia naturaleza"12. En la concepción filosófica de los integrantes de la Comisión Examinadora la persona humana goza de derechos pre-políticos que el orden constitucional no otorga, sino reconoce. Estos derechos, en virtud de la importancia que tienen en la constitución de la persona, son objeto de protección y reconocimiento a través del ejercicio de la resistencia. Su realización plena es el fin de la vida política y el objeto último al que debe aspirar el Estado Constitucional de Derecho. Toda ley que transgreda esos derechos y que sea extremadamente injusta no debe ser obedecida, sino resistida. Al respecto, es conocida la famosa formula de Radbruch (1999 [1946]) "la injusticia extrema no es derecho", que sirvió como argumento para condenar a los jerarcas nazis en los Juicios de Núremberg y que también utilizó el Tribunal Federal Alemán para castigar en 1992 a los guardianes del muro de Berlín que en la Alemania Democrática asesinaban, amparados por la ley, a los ciudadanos que intentaban cruzan la frontera. La doctrina de Radbruch que se adopta en esos fallos y que es defendida en la actualidad por autores de la filosofía del derecho como Vigo (2006) y Alexy (2001), destaca precisamente esa idea acerca de la existencia de un derecho supra-legal cuyo fundamento son los derechos esenciales del hombre y que, en virtud de su naturaleza, invalida el derecho vigente que sea contrario al principio de justicia. El derecho de resistencia toma su validez de ese plano meta-jurídico que se sustrae al campo del derecho positivo y tiene su norte en la protección de los derechos fundamentales del hombre.

\section{El valor de la resistencia débil en el contexto del Estado Democrático de Derecho actual}

Hemos dejado pendiente un problema que existe en relación con las formas de resistencia débil en el seno del Estado Constitucional de Derecho. La cuestión puede ser introducida mediante la siguiente pregunta: ¿por qué es necesario recurrir a la resistencia si la Constitución ya prevé formas y modalidades de "resistencia legal" en caso de violaciones a principios y normas constitucionales? En efecto, las constituciones modernas incorporan como mecanismo fundamental de protección de derechos el control de constitucionalidad de las leyes ordinarias en cabeza del Poder Judicial. Incluso más: si la sociedad se viera en la necesidad de incorporar de forma expresa nuevos derechos en la Constitución existe el proceso especial de modificación del texto.

Esta tesis, llevada a sus últimos extremos, nos conduciría a rechazar por completo la práctica de las formas de resistencia débil. En este sentido se

\footnotetext{
${ }^{12}$ Cf. Sampay (1995: 391).
} 
expresa Raz (1982: 327 y ss.), quien afirma que en las democracias la desobediencia civil es una conducta ilícita y moralmente reprobable, ya que se aparta de las vías de participación política y las formas convencionales para el control del ejercicio del poder y la reivindicación de los derechos ciudadanos. No todos piensan de la misma manera. Así por ejemplo, Rawls (2006: 347 y ss.), Habermas (1997: 56 y ss.) y Power (1972), entre otros, ubican a la desobediencia civil, en mayor o en medida, dentro de los valores políticos de la democracia: si bien es un acto ilegal, al ser público, consciente y no violento invita a la sociedad a reflexionar acerca de la justicia de las normas que se pretenden frustrar.

En relación con el problema planteado creemos que, si bien la Constitución provee mecanismos legales para la protección y el reconocimiento de derechos, la resistencia es necesaria y de indudable valor por dos razones. La primera de ella es de índole práctica. Muchas veces sucede que los medios legales establecidos en la ley no son eficaces a la hora de salvaguardar los derechos frente a su violación. Incluso muchas veces los ciudadanos o determinados grupos sociales (como las minorías culturales, por ejemplo) no tienen ni siquiera acceso a esos medios previstos por la ley. En todo caso, aunque los reclamos sean judicializadas, los procesos legales a veces suelen ser tan lentos que se convierten en mecanismos inservibles.

En realidad, quien viola abierta y deliberadamente la ley a través de las distintas formas de resistencia con el fin de preservar sus derechos esenciales, no deja de estar comprometido con el espíritu de la Constitución Democrática. La resistencia es un modo de expresar un compromiso fuerte con los principios y valores recogidos en ella. En efecto, el derecho, como idea práctica, indica un fin y un medio. Ese fin es la paz y el medio para alcanzar ese estado es la lucha en sus diversas manifestaciones. No se trata de cualquier lucha, por supuesto, sino de la lucha contra la injusticia. La lucha no debe pensarse como una característica ajena a la noción de derecho; la lucha es más bien una parte integrante de su existencia. El derecho es una idea de fuerza, de intereses que buscan ser escuchados y pretenden superar cualquier forma de opresión. En este sentido, aquel que se opone a las injusticias no actúa, en términos jurídicos, de manera rebelde, caprichosa o infundada. En un sentido profundo, en realidad, si el que sufre una injusticia se negara a actuar y no ejerciera la resistencia, estaría negando el derecho. Siempre que los derechos esenciales sean menoscabados, existe justificación suficiente como para rechazar la agresión, buscar hacer efectivo ese derecho y dejar en claro la importancia de su reconocimiento en el entramado social. Por lo demás, no es una cuestión menor destacar que quien defiende su derecho frente a una injusticia, defiende en última instancia también el interés que tiene toda la comunidad sobre él.

La segunda razón que justifica la resistencia radica en que las democracias actuales son imperfectas y los representantes populares no siempre tienen en cuenta los intereses de todos. Existen muchas voces que no son escuchadas. Todos tienen el derecho de que sus opiniones cuenten a la hora de la toma de decisiones políticas. Las formas de resistencia débil, como los movimientos de protesta, son muchas veces vehículos adecuados para que los grupos sociales y los individuos logren expresar sus intereses en el campo público. No 
podemos perder de vista, además, que las sociedades tienen una naturaleza cambiante. En el proceso de transformación y cambio político-social es posible que emerjan nuevas reivindicaciones de derechos. Muchos de los derechos que están plasmados en nuestra Constitución y en los instrumentos internaciones de Derechos Humanos fueron conquistas que derivaron de largas luchas. El derecho de huelga, por ejemplo, se consideró durante mucho tiempo ilegal; sin embargo, los trabajadores impulsaron su positivización a través de la resistencia y en la actualidad gran parte de los textos constitucionales lo reconocen como derecho fundamental. En los últimos años cabe poner como ejemplo en nuestro país el reconocimiento del matrimonio igualitario que lograron diferentes grupos sociales.

No podemos dejar de mencionar el reconocimiento de los derechos culturales frente al derecho estatal; en especial el derecho de los pueblo originarios. Se trata, por cierto, de un derecho que nace de sus propias prácticas, su tradición, valores y todo otro elemento cultural que define al grupo, aun cuando se oponga al sistema jurídico del Estado. El Estado no debe desconocer la praxis de estos grupos, sino tornar operativo su reconocimiento en armonía con la Constitución y la política internacional de los Derechos Humanos. Al respecto, se puede poner como ejemplo en caso del Sr. Crecencio Pilquiman, miembro de una comunidad mapuche de Chubut (Argentina), quien en el 2007 había demandado al Instituto Autárquico de Colonización (IAC) por haber adjudicado en venta a un estanciero una superficie aproximada de 2500 hectáreas en la cual se encontraba un cementerio de su pueblo. Si bien la solicitud de Pilquiman fue rechazada por el Tribunal Superior de Justicia de la Provincia del Chubut, el 7 de octubre de 2014 la Corte de Justicia de la Nación anuló la sentencia por entender que se "omitió absolutamente el tratamiento del planteo restante fundado en la vulneración del derecho a la consulta y participación de los pueblos indígenas, tutelado en el artículo 75, inc. 17 de la Constitución Nacional, así como en el Convenio 169 de la OIT".

Queda claro, entonces, que aun en el Estado Constitucional de Derecho no siempre las leyes se deben obedecer. También existen actos de resistencia permitidos y legítimos frente al derecho injusto o ante la necesidad de que sean reconocidos nuevos derechos que surjan de la dinámica propia de las sociedades y tengan su anclaje en los bienes humanos básicos del hombre. Frente a la imperfección de las democracias, la existencia de injusticas, la falta de representatividad y la ineficacia de los medios legales para proteger y garantizar los derechos esenciales, el derecho de resistencia se alza como un modo apropiado en la preservación de la integridad moral y social de la persona. Más que señal de abandono del orden democrático, la resistencia debe verse como una forma legítima de participación política en el interminable proceso de construcción de la democracia en el Estado de Derecho y en el reconocimiento de todas las voces en la esfera política.

\section{Conclusiones}

El análisis desplegado en estas páginas ha tratado de demostrar cómo la resistencia, lejos de ser una actitud transgresora, es un mecanismo esencial 
para preservación de los bienes humanos básicos de todos los ciudadanos, en especial, de aquellos grupos sociales que por diferentes cuestiones son relegados del campo público o no puede hacer valer con efectividad sus reclamos. En este sentido, no se puede desconocer la supremacía que tiene el derecho de resistencia en la transformación progresiva del derecho, ya sea para denunciar las injusticias o bien para reivindicar nuevos derechos. Vale aclarar, sin embargo, que la resistencia sólo es válida en la medida de que tienda a la defensa y protección de los derechos fundamentales del hombre en el marco del Estado Constitucional de Derecho.

El Estado Constitucional de Derecho, más allá de su valor, debe perfeccionarse, adecuarse a la realidad cambiante de la sociedad y aspirar a satisfacer los intereses de todos los involucrados en la empresa común. En esta tarea, el derecho de resistencia como genus juega un papel clave. En el caso de que los individuos o los grupos sociales no sean escuchados o sufran injusticias a causa de actos estatales o leyes que violen sus derechos más elementales, ellos tendrán derecho a ejercer cualquier forma de resistencia débil que consideren apropiada para presentar en la escena pública una causa que debe ser socialmente reconocida. Se trata entonces, de una forma de participación ciudadana y un modo de promover la intervención pública en un caso de injusticia o de reivindicación de derechos. Cuando se llegue a la instancia de que es el propio Estado de Derecho aquel que se encuentre en peligro, la sociedad no sólo tiene el derecho, sino el deber de ejercer la resistencia en su forma más extrema para defender el orden constitucional vigente de cualquier autoridad ilegítima que pretenda imponerse sobre él y la soberanía del pueblo.

\section{Bibliografía}

Agamben, G. (2007) Estado de excepción, Buenos Aires: Adriana Hidalgo. Alexy, R. (2001) "Una defensa de la fórmula de Radbruch", Anuario da Facultade de Dereito da Universidade da Coruña, $n^{\circ}$ 5, pp. 75-95.

Bobbio, N. (1997) El tercero ausente, Madrid: Cátedra. 
Carvajal, P. (1991) "Derecho de resistencia, derecho a la revolución y desobediencia civil en la temprana época moderna", Revista de Estudios Histórico-Jurídicos, pp. 241-273.

Dworkin, R. (1984) Los derechos en serio, Barcelona: Ariel.

Gargarella, R. (2007) "El derecho de resistencia en situaciones de carencia extrema", Astrolabio. Revista internacional de filosofía, $\mathrm{n}^{\circ}$ 3, pp. 1-29.

Gelli, M. A. (2008) Constitución de la Nación Argentina: comentada y concordada, tomo 1, Buenos Aires: La Ley.

Gramsci, A. (1974) Notas sobre Maquiavelo, la política y sobre el Estado moderno, Buenos Aires: Nueva Visión.

Habermas, J. (1997) "La desobediencia civil. Piedra de toque del Estado democrático de Derecho", en Ensayos políticos, Barcelona: Península, pp. 5171.

Kaufmann, A. (2006) Filosofía del derecho, Bogotá: Universidad Externado de Colombia.

Kelsen, H. 1949. Teoría General del Derecho y del Estado, México: Imprenta Universitaria.

Locke, J. (2006 [1689)] Segundo tratado sobre el Gobierno Civil. Un ensayo acerca del verdadero origen, alcance y fin del Gobierno Civil, Madrid: Tecnos.

Marx, C \& Engels, F. (1974 [1846]) La ideología alemana, Barcelona: Grijalbo.

Power, P. F. (1972) "Civil Disobedience as Functional Opposition", The Journal of Politics, vol. 34, $\mathrm{n}^{\circ} 1$, pp. 37-55.

Radbruch, G. (1999 [1946]) "Arbitrariedad legal y derecho supralegal", en Relativismo y derecho, Bogotá: Temis, pp. 25-42.

Rawls, J. (2006) Teoría de la justifica, México: F.C.E.

Raz, J. (1982) La autoridad del derecho. Ensayos sobre derecho y moral, México: UNAM.

Ross, A. (1961) Hacia una ciencia realista del derecho. Crítica del dualismo en el derecho, Buenos Aires: Abeledo-Perrot.

Sampay, A. E. (1995) Las Constituciones de la Argentina (1810-1972), Buenos Aires: EUDEBA.

Ugartemendia, J. I. (1999) "El derecho de resistencia y su constitucionalización", Revista de Estudios Políticos (Nueva Época), pp. 213245.

Vigo, R. (2006) La injusticia extrema no es derecho (De Radbruch a Alexy), Buenos Aires: La Ley.

Vitale, E. (2010) "Cambio político, constitución y derecho de resistencia", Isonomía, $\mathrm{n}^{\circ} 22$, 31-47. 